\title{
Habituation and dishabituation of rats' exploration of a novel environment
}

\author{
WILLIAM S. TERRY \\ University of North Carolina at Charlotte, Charlotte, North Carolina 28223
}

\begin{abstract}
The habituation of locomotor activity across repeated exposures to a novel maze was studied in a series of experiments using rats as subjects. Habituation, defined as a decrease in ambulation, was greater on a second trial occurring $5 \mathrm{~min}$ after a first trial than on one occurring 60 min after. This short-term decrement occurred only when the same maze was used on both trials, and could be dishabituated by intertrial detention in another novel environment. On a delayed test trial, habituation was, in one case, somewhat greater following initial spaced trials, and in another condition, comparable following both massed and spaced trials. The longer term habituation was maze specific, but was not affected by the presence of a dishabituator following either or both of the first two trials. The results were discussed in terms of theories of "priming" and encoding variability.
\end{abstract}

Much of the recent interest in the response decrement that occurs with repeated exposures to a stimulus (i.e., habituation) has focused on the short-term effects of prior stimulation. For example, Davis (1970) found more habituation of the startle response of rats during a sequence of massed tone presentations than during spaced presentations. Whitlow (1975), using a vasomotor component of the orienting response in rabbits, observed a short-term response decrement due to an immediately prior stimulus occurrence, that was separable from the longterm habituation due to the cumulative history of stimulations. Whitlow also found that the short-term decrement occurred only with repetitions of the same stimulus, and that it could be removed by placement of a dishabituating stimulus between repetitions of the target stimulus.

However, as Davis (1970) and Whitlow (1975) also noted, the long-term effects of such manipulations can differ from the short-term effects. For example, Davis found, on delayed test trials, that initial spacing of the stimulus presentations led to more habituation than did the initial massed presentations.

One theory of both the short- and long-term response decrements of habituation is Wagner's (1976) theory of priming in short-term memory (STM). According to this theory, a stimulus would be less effective in eliciting an unconditioned response if it occurred while a representation of that stimulus was

Portions of this research were presented at the Eastern Psychological Association's annual meeting, April 1978. This research was supported by funds from a Faculty Research Grant from the Foundation of the University of North Carolina at Charlotte and from the University of North Carolina. Address reprint requests to W. S. Terry, Department of Psychology, University of North Carolina at Charlotte, UNCC Station, Charlotte, North Carolina 28223. already active, or was "primed," in STM. Two methods are suggested by which a stimulus could be primed. "Self-generated" priming occurs through the persisting trace in STM of a prior presentation of that same stimulus. This form of priming would especially characterize the short-term response decrements observed in habituation (e.g., Davis, 1970; Whitlow, 1975). In "retrieval-generated" priming, a stimulus could be primed by other, associated cues, which act to retrieve representation of the target stimulus from long-term memory into STM. This form of priming would characterize the longer term response decrements observed by Davis (1970), such that contextual cues might be expected to become better associated to the habituating stimulus under conditions of spaced presentation, and thus would be better able to prime the target stimulus on subsequent occasions.

The present paper describes several experiments on habituation of exploratory behavior, using certain manipulations of both Davis' (1970) and Whitlow's (1975) experiments. While Davis included tests of the retention of habituation, neither the specificity of habituation nor dishabituation was assessed. And, while Whitlow did evaluate the effects of the latter two variables on short-term habituation, he did not assess their effects on delayed test trials. The present experiments, using a two-trial procedure, sought to determine (1) whether a short-term decrement in exploration occurred with successive exposures to a novel environment; (2) whether this effect was specific to repeated exposure to the same environment; and (3) whether the short-term response decrement could be reduced by exposure to a dishabituator. In all cases, a third test trial was used to assess the longer term effects of the prior manipulations.

A second major purpose of these studies was to 
provide an assessment of the usefulness of exploratory behavior as a paradigm for the study of habituation. Although some studies have used exploration to study selected aspects of habituation (e.g., Parsons, Fagan, \& Spear, 1973), little systematic work has been done to evaluate habituation of such behavior with respect to the several parametric features of habituation (Thompson \& Spencer, 1966). Since the present studies were intended as preliminary datagathering studies, relatively simple procedures and only one basic measure of exploration, the number of squares crossed per trial, were used instead of multiple measures of exploration as has been advocated (Fowler, 1965). However, as will be noted, these procedures yielded clear and unambiguous findings.

\section{EXPERIMENTS 1 AND 2}

The first two experiments sought to demonstrate a decrease in exploration of a novel maze as a function of just-prior vs. more-remotely prior exposure to the maze. Although there are many reports in the literature of decreases in exploratory activity with massed exposures to a stimulus (see Berlyne, 1960, for a review), some have not included spaced-trial comparisons to demonstrate the time dependency of the decrement (Montgomery, 1953). Experiments which have included such comparisons either have not separated the effects of a single, prior exposure to a stimulus from the cumulative effects of several prior massed trials (Danziger \& Mainland, 1954) or have not included remote tests of habituation to separate out short- from long-term response decrements (Berlyne, 1955). Finally, other studies have used relatively long intervals in the "massed" conditions, ranging from $2 \mathrm{~h}$ (Schneider \& Gross, 1965) to $12 \mathrm{~h}$ (Candland, Culbertson, \& Mayer, 1965).

The present studies compared 5- and 60-min intervals between two exposures to a maze. The longer interval was chosen to be shorter than the 24 -h intervals often found to produce little, or no, response decrement (e.g., Bronstein, 1972b; Montgomery, 1951, 1952). The 5-min interval was chosen because it was the shortest interval that could be used that would allow matching of several procedural details with the 60-min interval (e.g., intermixing trials of one subject with those of another; having each subject's trials immediately preceded by those of another; returning the subjects to the colony room during the delay and thus equating handling). A third, or test, trial was given either $1 \mathrm{~h}$ (Experiment 1) or $24 \mathrm{~h}$ (Experiment 2) after the second trial.

\section{Method}

Subjects. The subjects were 52 male Sprague-Dawley rats, approximately 55-70 days old. The subjects were received 5-7 days prior to the start of each study, and did not receive any special handling or gentling during this period. Each subject was individually housed and maintained on ad-lib food and water throughout the experiment. The colony room was maintained on a 12-h light-dark cycle, with subjects being run $3-6 \mathrm{~h}$ into the light cycle.

Apparatus. Exploratory activity was assessed in a black, U-shaped plywood maze. The interior dimensions were $15 \mathrm{~cm}$ wide $\times 19 \mathrm{~cm}$ high, with the lengths of the three stems being 60,100 , and $80 \mathrm{~cm}$. The interior of the maze was divided lengthwise into $1215 \times 20 \mathrm{~cm}$ blocks by fine yellow-painted lines, with three blocks in the first stem, five in the cross stem, and four in the last stem.

The maze was situated on a table in the middle of a large, open room. Illumination (approximately $646 \mathrm{~lx}$ ) was provided by overhead fluorescent lights.

Procedure. Subjects in each experiment were randomly assigned to one of two experimental groups ( $n s=14$ per group in Experiment 1, and 12 per group in Experiment 2). Each subject received three trials in the maze. For Group 5 in each experiment, the interval between Trial 1 and Trial 2 was $5 \mathrm{~min}$; for Group 60 , this interval was $60 \mathrm{~min}$. A third, test trial was given $1 \mathrm{~h}$ (Experiment 1 ) or $24 \mathrm{~h}$ (Experiment 2) after the completion of the second trial.

On each trial, the subject was individually carried in its home cage from the colony room to the testing room. The subject was then placed on the first square of the maze (the end square of the first stem) facing the back wall. The experimenter, standing behind the first stem of the maze, then started a clock and observed the subject for a 3-min period. The primary measure was the number of blocks crossed per minute, as counted by the experimenter and recorded on silent counters. A block was considered crossed if the subject's head and at least one foot crossed the boundary line leading to an adjacent block. Retracing or reentering a square produced a count only when all four feet were in the block being left. The experimenter also noted the most distant block reached in the maze during a trial, the maximum score here being 12 and the minimum 1 , and the number of boluses left in the maze.

Following completion of a trial, the subject was returned to the colony room, any boluses or urine was removed from the maze, and another subject (or several subjects, depending upon the length of the intertrial interval) was given a trial. After the appropriate delay, the first subject was returned to the testing room for its second or third trial, using the same procedures as before.

The order of running the animals of Groups 5 and 60 evenly distributed subjects of the two groups across the experimental session. In addition, subjects in each group were about equally often preceded in the maze by a subject from the same group, as from the opposite group. No special procedures, other than the above, were used to minimize potential odor trails. Each subject's trials were immediately preceded by those of another subject, thus presumably equating such cues. Prior to running the first subject in a session, and whenever a long interval intervened between trials, a nonexperimental animal was given a maze exposure, thereby holding constant just-prior maze occupancy for all subjects.

\section{Results}

Locomotor activity within trials generally showed a high rate of block crossings during the 1st minute, which then declined during the 2 nd and 3 rd minutes of each trial. For example, the mean number of squares crossed on successive minutes of Trial 1 were $15.5,10.8$, and 11.4 when combined over all subjects in these two experiments. This finding is consistent with prior observations of within-trial decrements 


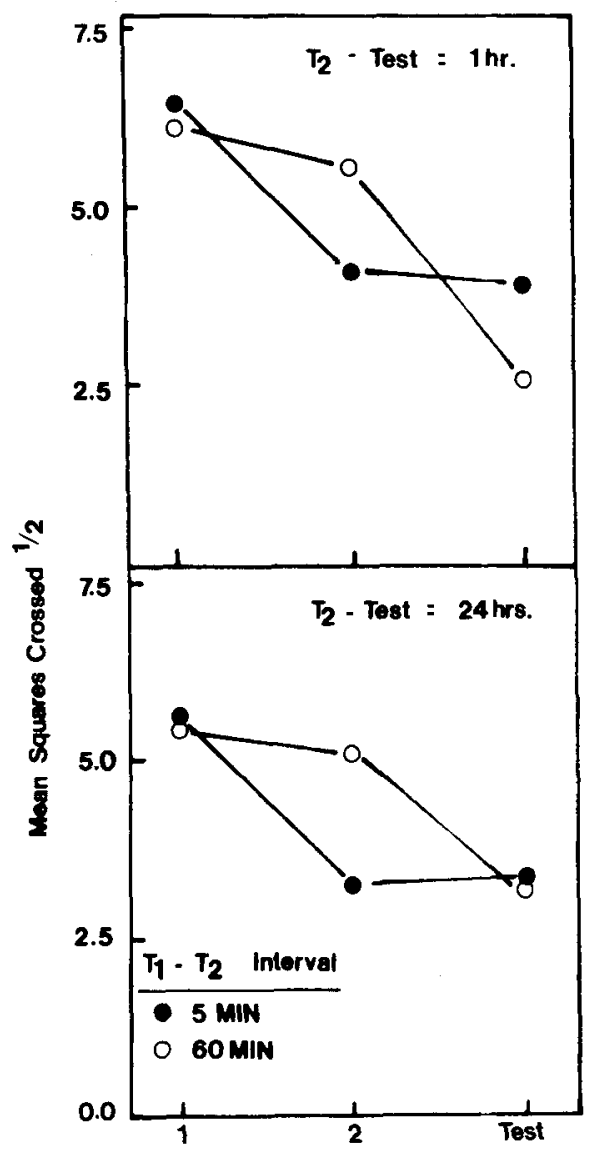

TRIALS

Figure 1. Mean transformed number of blocks crossed over trials, for groups having a Trial 1-Trial 2 interval of 5 or 60 min. The upper panel shows the results of Experiment 1 in which the delay of test interval was $1 \mathrm{~h}$; the lower panel shows the results of Experiment 2 in which the delay of test interval was $24 \mathrm{~h}$.

in exploratory behavior (e.g., Bronstein, 1972a, 1972b). Since there were no systematic effects of within-trial activity among the several groups in these studies, the locomotor activity data will be presented in terms of the total number of crossings per trial.

Due to the large amount of variability in individual activity scores, the counts were subjected to a square root transformation. The results of Experiment 1 are shown in the upper panel of Figure 1, which plots mean transformed number of blocks crossed across the three trials for groups of subjects receiving either a 5- or a 60-min Trial 1-Trial 2 interval. While both groups showed comparable levels of activity on Trial 1, subjects in Group 5 showed a large decrease in activity on Trial 2, relative to subjects in Group 60. However, on the test trial given $1 \mathrm{~h}$ later, Group 60 subjects were less active than Group 5 subjects.' Thus, more short-term habituation was observed on a massed repetition of the maze exposure, while slightly greater long-term habituation was observed following spaced training.
An analysis of variance of the data supported the above observations, with a reliable Groups (5 vs. 60$)$ by Trials $(1,2$, test $)$ interaction $[F(2,52)=5.98$, $\mathrm{p}<.05]$. Post hoc $t$ tests, using error terms from the analysis of variance, showed that Groups 5 and 60 did not differ in their levels of activity on Trial 1 $(\mathrm{t}=.56)$; Group 5 crossed fewer blocks on Trial 2 than did Group $60[\mathrm{t}(39)=2.08, \mathrm{p}<.05]$; and Groups 5 and 60 did not differ significantly on the test trial $[\mathrm{t}(39)=1.86, \mathrm{p}<.10]$.

Further analyses of the Groups by Trials interaction showed that only Group 5 evidenced a significant decline in activity from Trial 1 to Trial 2 [t(52) = $4.12, \mathrm{p}<.01]$, while only Group 60 showed a decline between Trial 2 and the test trial $[\mathrm{t}(52)=5.11$, $\mathrm{p}<.01]$.

The locomotor activity results of Experiment 2, in which the test trial occurred $24 \mathrm{~h}$ later, are shown in the lower panel of Figure 1. The data on the first two trials replicate the results of Experiment 1, with a substantial decrease in activity only for subjects in Group 5. However, subjects in Group 60 did evidence a decline in activity from Trial 2 to the test trial, to a level comparable to that of Group 5. Statistical analysis showed a nonsignificant Groups by Trials interaction $[\mathrm{F}(2,44)=2.90, \mathrm{p}<.10]$. However, while Groups 5 and 60 did not differ significantly on either of Trials 1 or the test trial (ts $<1$ ), there was significantly less activity on Trial 2 for subjects in Group 5 than for subjects in Group 60 [t(33) = $2.06, \mathrm{p}<.05$ ]. In comparisons across trials, only Group 5 showed a reliable decline in activity between Trials 1 and $2[\mathrm{t}(44)=3.54, \mathrm{p}<.01]$, and only Group 60 showed a decline between Trial 2 and the test trial $[t(44)=2.77, p<.01]$. Thus, in this second experiment, a massed repetition again produced more habituation during the repeated stimulus presentation, with this superiority disappearing on the delayed test.

One trend which is not apparent in Figure 1 is the occurrence of recovery from habituation between trials. Analysis of the minute-by-minute counts showed that recovery consistently occurred between the end of one trial and the start of the next whenever the interval between trials was $1 \mathrm{~h}$ or longer. For example, the mean number of blocks crossed during the first minute of Trials 1 and 2 were equivalent for subjects in the 60-min groups. Recovery at the start of the test trial was not as complete, however, and, of course, lower levels of activity were reached during Trial 2 and the test trial than during Trial 1.

While the above block-crossing data measures the overall level of activity, it does not describe whether all or only part of the maze was explored on each trial. However, the measure of the maximum distance traversed into the maze revealed a pattern of responding similar to that shown in Figure 1. Thus, over both experiments, Groups 5 and 60 had com- 
parable mean distances on Trial 1 (10.46 and 9.92, respectively) and the test trial (7.58 and 6.42$)$, with Group 60 going further into the maze on Trial 2 (10.38 and 6.61). The Groups by Trials interaction of this data was highly significant $[\mathrm{F}(2,100)=7.93$, $\mathrm{p}<.01]$.

While it is possible that the reduced activity over trials was due to an increase in fear being manifested, the measure of defecation in the maze does not fully support this interpretation. In Experiment 1, there was no increase in the number of boluses over trials (overall mean $=1.24$ ) and no between-group differences on any trial. In Experiment 2, there was an increase in boluses from Trials 1 and 2 to the test trial (means $=1.29$ and 3.25 , respectively), which was significant $[F(2,44)=7.82, p<.01]$. Again, Groups 5 and 60 did not differ on any of the trials.

A further analysis of the fear-activity relationship, using point-biserial correlations between the ambulation scores and the presence or absence of boluses on each trial, also failed to reveal any systematic effects. In Experiment 1, the correlations were all smal (range: -.03 to -.15 ). In Experiment 2, the correlations on each trial were somewhat larger and consistently negative (range: -.25 to -.37 ), but none reached statistical reliability, and there were not substantial intertrial differences in the coefficients.

It has sometimes been noted (e.g., Collerain, 1978; Dember \& Kleinman, 1973) that an animal's behavior in a maze is influenced by odor trails left by previous subjects run in the same apparatus. Two sets of analyses of the present ambulation data assessed such potential effects here. One analysis involved computation of activity scores separately for subjects immediately preceded by another subject of the same group or by a subject of the opposite group. The pattern of results of Figure 1 was exactly reproduced within these two subgroups of Groups 5 and 60, with no apparent effect on one subject's activity of the experimental condition of the preceding subject. A second analysis assessed the correlation between each subject's activity score and the score of the subject run immediately prior in the maze. This correlation was essentially zero on each trial. These two analyses thus give some assurance that the locomotor activity observed was not contaminated by odor trails or other factors due to the preceding subject in the maze.

\section{Discussion}

Several features of the present results are worth noting. First, a locomotor response decrement occurred with repeated stimulation, and recovery from the decrement took place with the longer intervals between stimulations. Of more importance, there was a short-term decrement in activity with repeated exposure to the maze separate from the long-term decrement. This finding alone is not surprising, and is in agreement with other reports in the literature. The present observations are also consistent with a number of the parametric characteristics of habituation described by Thompson and Spencer (1966).

However, the massed-trial decrement must be qualified in the present case. On the third, test trial, response suppression with initial spaced stimulation was slightly more than (Experiment 1 ) or equal to (Experiment 2) response suppression with initial massed stimulation. Taking the two experiments together, at the very least it can be acknowledged that spaced trials produce comparable long-term habituation to massed trials in the present situation.

One straightforward interpretation of the increase in habituation on the test trials with spaced repetitions would be that subjects in this condition had much greater total exposure to the maze. Both on the overall activity measure and on the maximum distance measure, subjects in Group 60 were exposed to much more of the maze on Trial 2 than were subjects in Group 5. Thus, it may not be surprising that the Group 60 subjects showed substantial response decrements on the test trial.

What then becomes of interest is the fact that subjects in Group 5 also showed so much suppression on the test trial, even though less exposure had occurred to the whole maze on the prior trials. This may be due to qualitatively different exploratory reactions of subjects in the two groups on Trial 2: recognition of the maze as familiar by Group 5 , accompanied by more intensive investigation of the first blocks of the maze, vs. recognition of the maze as less familiar by Group 60, accompanied by more intensive investigation of the whole maze (Fowler, 1965; Glanzer, 1961). However, each tendency may ultimately have had the same effect of producing recognition of the maze, thus leading to comparable behavior on the test trial.

The bolus measure failed to show a trial-spacing effect in the present studies, although other research has reported such effects in comparing 12-h and 7-day intertrial intervals (Candland et al., 1965). The pronounced increase in the number of boluses on the test trial of Experiment 2 could have indicated either the development or the appearance of fear, which then acted to suppress locomotor activity and mask potential differences due to the spacing manipulation. However, the earlier described analyses failed to give any strong support to a fear-activity relationship.

\section{EXPERIMENT 3}

One major finding of the first two experiments was a short-term decrement in locomotor activity following a single prior exposure to the maze. The next 
experiments investigated some of the conditions responsible for the short-term effect. Experiment 3 assessed the stimulus specificity of the decrement. As noted earlier, Whitlow (1975) found the short-term habituation to occur only with repetitions of the same stimulus. In a study of exploratory behavior, Montgomery (1953) demonstrated a generalization function among mazes differing only in color, with the second-trial decrement being smaller the greater the discrepancy in color from the first trial maze. No remote tests of habituation were reported, however.

The present study varied the shape of the mazes across Trials 1 and 2. Two mazes were therefore used, the U-shaped maze and a smaller, T-shaped maze. No attempt was made to equate the mazes in terms of initial response tendencies elicited, rate of habituation in each, etc. Clearly, this task alone would have required a major investigation. The present purpose was simply to determine whether the short-term response decrements observed in the present series of studies were due specifically to prior exposure to the same maze, or were due to incidental aspects of the experimental procedures such as recent handling of the animals, response fatigue, or prior exposure to any novel environment. Therefore, subjects were exposed to either the same maze on both Trial 1 and Trial 2, or to different mazes on Trials 1 and 2 . In addition, a test trial given $24 \mathrm{~h}$ later in the same maze as Trial 2 similarly assessed whether the long-term decrement was specifically due to two prior exposures to the same maze.

\section{Method}

Except where noted, the subject characteristics, apparatus, and procedures were like those of the first two experiments.

In addition to the $U$-maze, a $T$-shaped maze was used, having interior dimensions of $10.2 \mathrm{~cm}$ wide $\times 15 \mathrm{~cm}$ high, with the stem length being $59.5 \mathrm{~cm}$ and each of the arms $61.7 \mathrm{~cm}$. The maze was made of plywood, painted flat black, and divided into nine $10.2 \times 20 \mathrm{~cm}$ blocks by yellow boundary lines.

During the first session, experimentally naive subjects were given two maze trials, separated by a 5 -min intertrial interval. For subjects in Group Same $(n=14)$, the same maze was used on both trials, this being the U-maze for half the subjects and the $T$-maze for the remaining subjects. For subjects in Group Different $(n=14)$, a different maze was used on Trials 1 and 2 , these being the U-maze followed by the $T$-maze for half the subjects and the reverse sequence for the remaining subjects. During the intertrial interval, the subjects were returned to the colony room in their home cages while another subject received its trial, just as in the first two experiments.

On the test trial given $24 \mathrm{~h}$ later, each subject was placed in the same maze as it had been exposed to on Trial 2.

The order of running animals intermixed subjects from the two groups, and trials with nonexperimental animals were run whenever a substantial delay occurred between trials in a particular maze.

\section{Results and Discussion}

Although the T- and U-mazes differed physically in a number of characteristics, measures of over- all locomotor activity were comparable in them. On Trial 1, the mean transformed number of blocks crossed was 6.58 in the U-maze and 6.51 in the T-maze. Since performance was similar (except where noted below) over trials between subgroups run in the different mazes, the data were pooled across mazes.

In Figure 2 are shown the mean transformed number of blocks crossed per trial for the two experimental groups. The first point to note is that there was a decrease in activity on Trial 2 by subjects in Group Sam: This decrease was statistically significant $[t(13)=3.78, p<.01]$ and replicates the shortterm decrement observed in the first two experiments. Subjects in Group Different showed, if anything, slightly more activity on Trial 2 , though this increase was not statistically significant $(t=.88)$.

The comparisons of primary interest are between Groups Same and Different on Trial 2 and the test trial. As can be seen in Figure 2, subjects in Group Different were much more active than those in Group Same on Trial 2, and, to a lesser extent, on the test trial. Thus, the short-term decrease does not appear to be due to recent exposure to any novel stimulus or to factors incidental to the procedures of conducting a prior trial. In addition, the long-term decrease appears to be due to differences in the prior manipulations.

The above observations were supported by the results of a mixed-factorial analysis of variance, using as between-groups factors the two groups of subjects and the two mazes, and the within-subjects

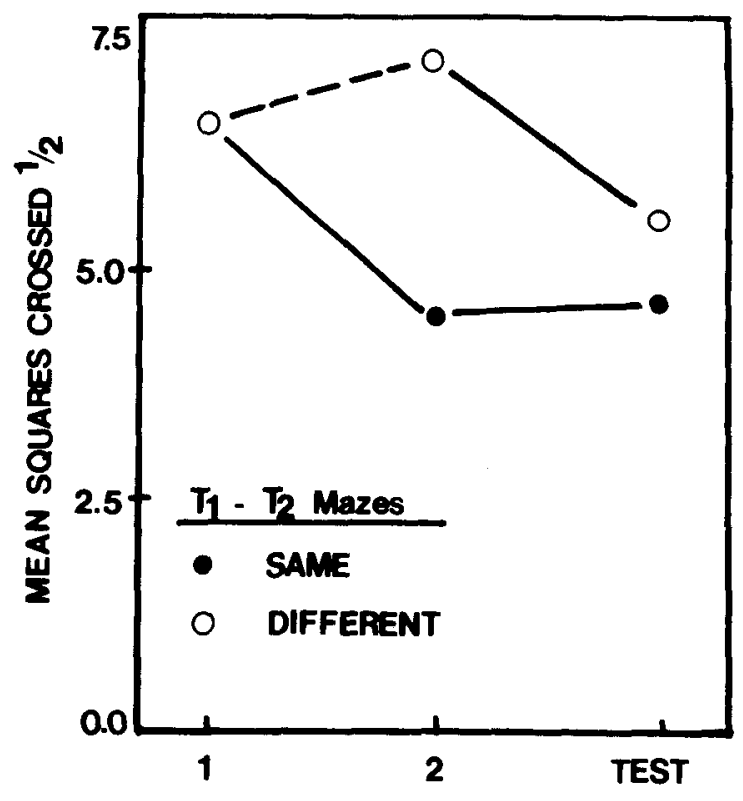

TRIALS

Figure 2. Mean transformed number of blocks crossed over trials for groups run in the Same maze on all trials or in a Different maze on Trial 1 in Experiment 3. 
factor of trials (comparing only Trial 2 and the test trial, when subjects were run in the same maze). Aside from the main effects of Groups $[\mathrm{F}(1,24)=$ $13.90, p<.01]$ and of Trials $[F(1,24)=8.57, p<$ $.05]$, there was a significant Groups by Trials interaction $[F(1,24)=13.49, p<.01]$. Activity did not change from Trial 2 to the test trial for subjects in Group Same $[\mathrm{t}(24)=.52]$, though there was a decrease in activity for subjects in Group Different $[\mathrm{t}(24)=4.67, \mathrm{p}<.01]$.

There was also found to be a Groups by Maze by Trials interaction $[F(1,24)=5.53, p<.05]$. Post hoc tests indicated that, in both mazes, Group Same was less active than Group Different on Trial $2[\mathrm{t}(24)=$ $3.53, \mathrm{p}<.01]$. On the test trial, only subjects run in the U-maze showed less activity in the Same condition as opposed to the Different condition, the mean scores being 3.70 and 5.35 , respectively $[\mathrm{t}(24)=2.06$, $\mathrm{p}<.05]$. Subjects in the Same and Different groups run in the $\mathrm{T}$-maze showed a convergence in their levels of activity on this trial (means $=5.57$ and 5.81 , respectively); this may have been due to the smaller size of the T-maze, leading to more habituation per trial there than in the U-maze.

While the $\mathrm{T}$ - and $\mathrm{U}$-mazes generated comparable numbers of block crossings, the two mazes were differentiated in terms of the measure of maximum distance traversed into the maze. All subjects run in the $\mathrm{T}$-maze crossed each of the nine blocks on every trial. Subjects run in the U-maze, on the other hand, showed a pattern of behavior like that of the overall block crossing measure.

Groups Same and Different did not differ in the mean number of boluses left on each trial, and there were no between-maze differences on this measure. There was an overall increase in the number of boluses from Trials 1 and 2 to the test trial (means $=.81$ and 1.97 , respectively $[F(1,24)=19.13, \mathrm{p}<.01]$, replicating the results of Experiment 2.

The present results indicate that the short-term response decrement in locomotor activity is due to recent, prior exposure to the same maze. The test trial data also indicate, at least for subjects run in the U-maze, that response suppression was greater following two exposures to the tested maze, as compared with one exposure to it and another exposure to a different maze.

Even though the amount of fear, as indicated by the number of boluses, significantly increased on the test trial, the Same-Different difference in activity remained, arguing that any fear-produced response suppression was not sufficient to mask completely effects due to the primary manipulation. The comparability of the two groups on the bolus measure suggests that it was less sensitive to the specific mazes previously explored than to more general features of the experimental procedures, such as handling.
The present locomotor activity data confirm Montgomery's (1953) findings of stimulus specific decrements in exploration occurring with closely spaced trials. They are also consistent with another of Thompson and Spencer's (1966) characteristics of habituation, that a generalization decrement occurs to other test stimuli. Recent studies have affirmed the specificity of both short-term (Whitlow, 1975) and long-term (Rubel \& Rosenthal, 1975) habituation.

\section{EXPERIMENT 4}

The next experiment investigated whether the short-term response decrement could be reduced by exposing the subject to a novel, dishabituating stimulus between Trials 1 and 2. Few observations of dishabituation of exploratory behavior are available in the literature. Terry (1978) found that detainment in a novel container between exposures to a T-maze disrupted spontaneous alternation behavior. Thomas (1974) observed that intertrial exposure to a novel environment reduced habituation to the preexposed environment, using a resident-intruder paradigm.

In the present experiment, the dishabituating stimulus consisted of placing the subjects in a novel enclosure for part of the 5-min Trial 1-Trial 2 interval, rather than in a familiar enclosure (the home cage) as had been done in the preceding experiments. A remote test trial was used to determine the longer term effect of the dishabituation manipulation. A control experiment assessed whether exposure to the novel enclosure in the absence of prior maze testing had any effects (e.g., sensitization or fear arousal) on subsequent locomotor activity.

A second purpose of this study was to determine the degree of response decrement on a delayed test trial following a single prior exposure to the maze. Experiments 2 and 3 demonstrated a maintained activity decrement between the two sessions of the experiments if subjects were tested in the same maze on all trials. It is possible that such a decrement might not reflect the cumulative effects of two prior exposures, but rather some other process (e.g., incubation of fear) requiring only a single prior trial.

\section{Method}

Thirty-six experimentally naive rats, similar to those previously used, were randomly assigned to three equal-sized groups. Two groups were given two trials in the U-shaped maze on Day 1 , separated by a 5 -min intertrial interval. Subjects in Group Home Cage spent this interval in their home cages in the colony room, as was the case in the prior studies. Subjects in Group Novel Box were returned to the colony room in their home cages after Trial 1 (this taking about $45 \mathrm{sec}$ ), and then were transferred to a novel container for approximately $3 \mathrm{~min}$. This container was a black Plexiglas (sides and top) box with a grid floor, and having interior dimensions of $10.3 \times 19.5 \times 20.5 \mathrm{~cm}$. At the end of the detention interval, the subjects were returned to their home cages and brought to the test room for Trial 2. Finally, subjects in 
Group No-T2 did not receive a second trial on Day 1, but rather were immediately returned to the colony room in their home cages following Trial 1. Twenty-four hours later, subjects in all three groups received a test trial in the maze.

After this experiment was completed, a control experiment was conducted to evaluate possible effects of exposure to the novel container alone on activity. Two additional groups of naive subjects were formed. Subjects in one group $(n=9)$ were given a 3-min exposure to the novel box prior to a single trial in the maze, while subjects in a second group $(\mathrm{n}=13)$ were simply given a single maze exposure.

\section{Results and Discussion}

The results of the control experiment showed there was no effect on activity during a single maze exposure of just-prior placement in the novel container. The mean transformed number of squares crossed were 6.28 for subjects held in the novel box and 5.70 for subjects not placed in the box; this difference was not significant $(t=.88)$. This finding is consistent with previous reports that extramaze exposures to loud noises or electric shocks have no appreciable effects on subsequent exploration (Montgomery \& Monkman, 1955).

The main results of this study are shown in Figure 3, which plots mean transformed activity scores across the three trials of the experiment. The first point to consider is the effect of the dishabituating stimulus on activity during a closely following second trial. As can be seen in Figure 3, subjects that spend the intertrial interval in a familiar environment (i.e., Group Home Cage) show the usual decrease in activity on Trial 2, while subjects that spend the intertrial

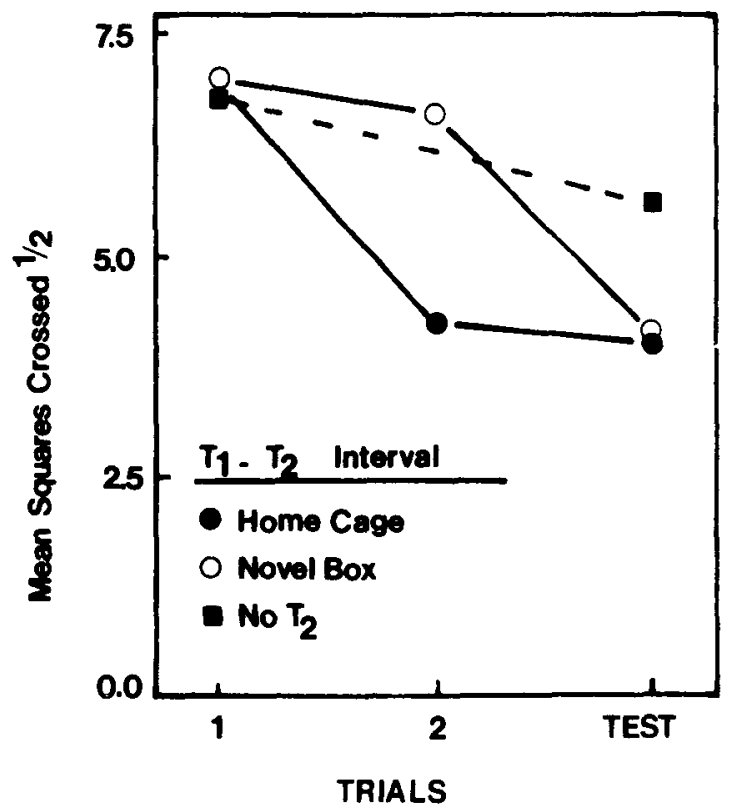

Figure 3. Mean transformed number of blocks crossed over trials for groups detained either in their home cages or in a novel box during the Trial 1-Trial 2 interval, and for control subjects not receiving a Trial 2, in Experiment 4. interval in a novel environment (i.e., Group Novel Box) show no such decrease.

When habituation was assessed on the test trial 24 h later, Group Home Cage showed little change from the low level of activity of Trial 2. However, the dishabituated Group Novel Box showed a sharp decrease in activity, and was not substantially different from Group Home Cage. Thus, while the novel box exposure reduced the short-term response decrement, it had no effect on the longer term habituation observed.

A Groups by Trials analysis of variance of these data indicated a significant interaction between the two variables $[F(2,44)=4.47, p<.05]$. Post hoc tests indicated that the decrease in activity from Trial 1 to Trial 2 was reliable only for subjects in Group Home Cage [t(44) $=4.35$ ], and that the decrease in activity from Trial 2 to the test trial was reliable only for subjects in Group Novel Box [t(44) $=4.04$, ps $<.011$. There was also a significant difference between Groups Home Cage and Novel Box on Trial $2[t(33)=2.89, p<.01]$, but not on either Trial 1 or the test trial.

The second question this study addressed was whether the observed levels of performance on the test trial depended upon two prior exposures to the maze or only one. The results of Group No-T2 are shown in Figure 3, which plots performance of this group of subjects on Trial 1 and the test trial $24 \mathrm{~h}$ later. In comparison with the other two groups, the level of activity was similar on Trial 1 and decreased on the test trial, though not to the low level of the other groups. Therefore, the delayed test trial was sensitive to the cumulative effects of two prior trials.

A Groups (Home Cage, Novel Box, No-T2) by Trials ( 1 and test) analysis showed a significant interaction of these two variables $[\mathrm{F}(2,33)=3.32$, $\mathrm{p}<$ $.05]$. Post hoc tests indicated that Groups Home Cage and Novel Box had lower levels of activity on the test trial than did Group No-T2 $[\operatorname{ts}(33)=2.14$ and 2.04 , respectively, ps $<.05]$. The decrease in activity from Trial 1 to the test trial was only marginally significant for Group No-T2 [t(33) $=1.99$, $\mathrm{p}<.10]$.

The alternate response measures for these three groups showed results consistent with the previous experiments. Thus, the mean maximum distance measure again paralleled the overall activity measure as shown in Figure 3, while the bolus measure showed an increase from the 1st day's trial(s) to the test trial with no between-groups differences. Of some interest is the fact that exposure to the novel box did not seem to increase fear, at least in terms of the number of boluses left in the maze on the subsequent trial.

The present results thus serve to demonstrate yet another characteristic of habituation of exploratory 
activity, that of dishabituation (Thompson \& Spencer, 1966). The equivalent long-term habituation observed in subjects exposed to the novel box, or not, suggests a transient effect of the dishabituator influencing immediately subsequent activity but not the overall level of habituation attained.

\section{EXPERIMENT 5}

In Experiment 4, it was found that exposure to a novel environment during the Trial 1-Trial 2 interval blocked the appearance of the short-term response decrement while having no effect on the longer term habituation observed. The present experiment sought to determine whether exposure to the novel environment following both Trial 1 and Trial 2 would prevent the long-term decrement from occurring. Whitlow and Wagner (Wagner, 1976), using the vasomotor response of rabbits, found that presentation of a dishabituator after each occurrence of the to-behabituated stimulus reduced long-term habituation to this stimulus. This result was consistent with their view that the dishabituator was disrupting the posttrial rehearsal necessary to produce long-term memory for the target stimulus. Similarly, it could be questioned whether the novel environment here simply masked the presence of habituation (as via a sensitization process) or prevented the occurrence of habituation altogether (as via a posttrial disruption of short-term memory). Three groups of subjects were run in this study, differing in whether they received a novel-box placement following Trial 1 only, Trial 2 only, or both Trials 1 and 2 .

\section{Method}

The apparatus and procedures were similar to that of the prior experiment. Experimentally naive subjects were assigned to one of three groups, differentiated by their experimental treatments on Day 1. Subjects in Group After T1 $(n=16)$ were placed in the novel box only following Trial 1 in the U-maze and were returned to the colony room in their home cages following Trial 2. Group After $\mathrm{T} 2(\mathrm{n}=10)$ were placed in their home cages following Trial 1, and then placed in the novel box following Trial 2 . For half the subjects in each of these groups, the black Plexiglas box of the previous experiment was used as the novel box; for the other half a white box, otherwise identical to the black one, was used. Finally, subjects in Group After T1 \& T2 $(n=16)$ were placed in the novel box following both Trial 1 and Trial 2, and after the second placement were returned to their home cages in the colony room. In order to maintain the novelty of the dishabituating stimulus, these subjects were placed either in the black box following Trial 1 and the white box following Trial 2, or vice versa. Subjects in all three groups were given a test trial $24 \mathrm{~h}$ later.

\section{Results and Discussion}

The mean transformed locomotor activity scores from Experiment 5 are shown in Figure 4. Several points should be noted. First, subjects not exposed to the novel box between Trials 1 and 2 (Group After T2)

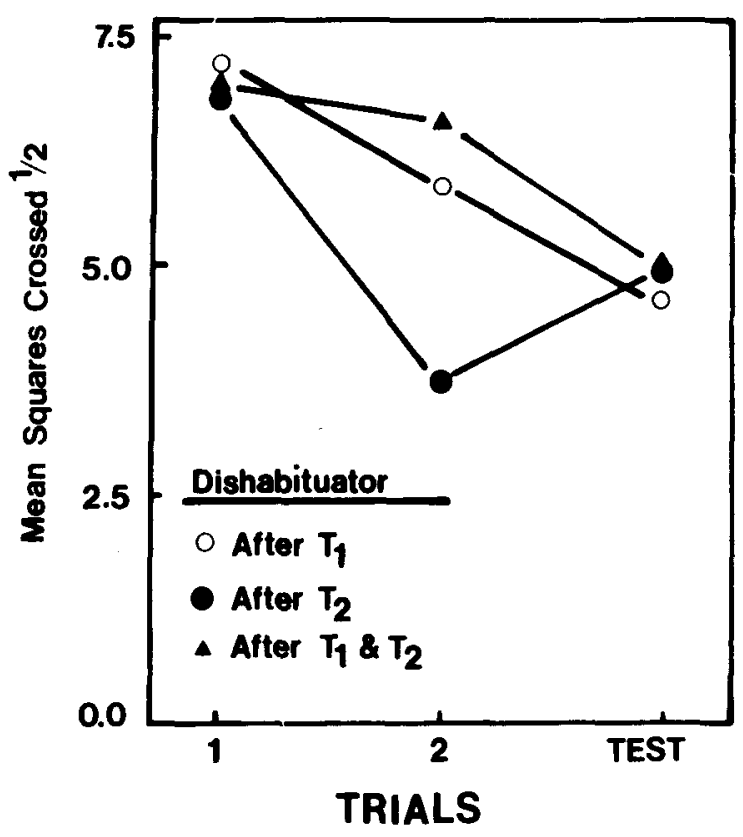

Figure 4. Mean transformed number of blocks crossed per trial for groups detained in a novel box after Trial 1 only, after Trial 2 only, or after both Trials 1 and 2, in Experiment 5.

showed the usual substantial decrease in activity on the latter trial. Second, subjects placed in the novel environment between these trials (Groups After T1 and After T1 \& T2) showed little, if any, such decrease on Trial 2. Thus, the dishabituation effect of the preceding experiment was reproduced. Finally, a second placement in a novel environment following Trial 2 (Group After T1 \& T2) did not interfere with the long-term habituation observed on the test trial, as all three groups performed comparably at this time.

Statistically, Group After T2 showed a decrease in activity over trials $[\mathrm{F}(2,27)=4.14, \mathrm{p}<.05]$, with significantly less activity on Trial $2(t=2.86)$ and the test trial $[\mathrm{t}(1.73, \mathrm{p}<.10]$ than on Trial 1 . The rise in activity between Trial 2 and the test was not significant $(t=1.12)$. In addition, on Trial 2 these subjects not exposed to the dishabituator were less active than the combined groups of subjects exposed to it prior to Trial $2[\mathrm{t}(40)=3.03, \mathrm{p}<.01]$.

For those two groups of subjects exposed to the novel box between Trials 1 and 2, there was an overall decline in activity across trials $[\mathrm{F}(2,60)=16.59$, $\mathrm{p}<.01$ ]. The change from Trial 1 to Trial 2 was not reliable $(t=1.47)$, although it was from Trial 2 to the test trial $[\mathrm{t}(60)=2.55, \mathrm{p}<.02]$.

The distance and bolus measures revealed results comparable to those of the prior experiments, and thus need not be discussed in detail. The primary findings were less exploration of the whole maze on Trial 2 by subjects not just exposed to the novel box and for all groups on the test trial, and an overall increase in the number of boluses on the final trial. 
The results of this experiment again indicate that the novel-box dishabituating stimulus acts to disrupt the appearance of short-term habituation but has no effect on the longer term habituation. The results do not support the view that the dishabituator was acting to disrupt rehearsal and therefore also habituation, at least in the present situation. This appears reasonable in light of the rather long intervals used between exposure to the maze and placement in the dishabituating environment. It is possible that any, or most, posttrial processing that occurred was completed by the time the dishabituation procedure began.

\section{EXPERIMENT 6}

The results of Experiments 4 and 5 suggest that the dishabituator was only effective in blocking shortterm habituation, having no effect on longer term habituation. So far it has been argued that the shortterm effect is due to influences of the dishabituator on processing of the preceding maze exposure (e.g., displacing representation of the latter from STM). It is also possible that the dishabituator had a proactive effect (e.g., sensitization) on performance during the subsequent trial. While the control study found no evidence for such effects in unhabituated animals, this may have been due to the already high rate of activity on the first exposure to the maze. In the present study, the novel-box exposure occurred prior to the test trial in order to assess its effects on a lower activity baseline and in the absence of a shortly prior maze exposure. A sensitization hypothesis would predict response enhancement on this last trial following the novel-box placement. The notions of priming and dishabituation would not make this prediction, since short-term habituation is not being affected.

\section{Method}

During the first session, 24 naive subjects were given two exposures to the maze separated by a 5 -min interval. The animals were then assigned to two groups equated on their performance on these trials. Twenty-four hours later, a test trial was given. Subjects in Group Novel Box were placed in the black Plexiglas novel box $5 \mathrm{~min}$ before the third trial; subjects in Group Home Cage simply received the test trial.

\section{Results and Discussion}

The mean transformed number of squares crossed on Day 1 for Groups Home Cage and Novel Box, respectively, were: 7.09 and 6.83 on Trial 1 , and 5.28 and 5.17 on Trial 2. On the test trial, subjects in Group Home Cage had a mean score of 4.19 , and subjects in Group Novel Box, 5.48. Thus, performance was somewhat higher just following exposure to the novel box, although the difference was not statistically reliable. The Groups by Trials interaction was not significant $[F(2,44)=2.22, p>.10]$, as well as the results of a separate $t$ test on the test trial data $(t=1.45)$.

The only reliable effect was an overall decrease in responding across trials $[F(2,44)=15.06, p<.01]$. Post hoc tests indicated that the decrease was significant between Trials 1 and $2[\mathrm{t}(44)=3.16]$ and between Trial 1 and the test trial $[\mathrm{t}(44)=3.54$, ps $<$ .01 ], but not between Trial 2 and test. This same pattern of significant ef fects held within each group.

The results of Experiments 4-6 indicate that the novel-box placement did not sensitize subsequent activity, but instead suggest that the dishabituator was only effective in reducing short-term habituation due to a recent presentation of the target stimulus. The dishabituator apparently had little effect on long-term habituation, either when presented during the habituating phase or just prior to testing.

\section{GENERAL DISCUSSION}

The short-term activity results of the present experiments are in agreement with several of the general characteristics of habituation described by Thompson and Spencer (1966). Thus, with short intertrial intervals, locomotor activity decreased during the second exposure to the maze, and with longer intervals, there was recovery of activity from the end of one trial to the start of the next (Experiments 1 and 2). The decrement was stimulus specific (Experiment 3), and was disrupted by exposure to another, dishabituating stimulus (Experiments 4 and 5). These findings thus argue that the response decrements were not due to sensory adaptation or response fatigue, which are frequently cited alternatives to habituation.

The interpretation of the short-term activity decrements must be tempered by the argument that, in fact, possibly only one aspect of the exploratory tendency may have shown habituation, that of exploring the entire maze. As has been noted (e.g., Fowler, 1965), a reduction in gross ambulation scores may simply reflect more intensive exploration of restricted portions of the maze.

A somewhat different pattern of results was found on the test trials. Here, initial spaced exposures produced at least equal response decrement to initial massed exposures (Experiments 1 and 2). The presence of a dishabituating stimulus following either or both of Trials 1 and 2 (Experiments 4 and 5) or prior to the test trial (Experiment 6) had no significant effect on long-term habituation, as response decrements were substantial and comparable to those of subjects not exposed to the novel box. However, the delayed tests were sensitive to the maze specificity of the response decrement (Experiment 3) and to the number of trials (Experiment 4). 
Several interpretations of the different short- vs. long-term effects are possible. It could be that, while overall retention was equal as a function of the combined Trial 1-Trial 2 manipulations, various aspects of training contributed differentially to such retention (e.g., differential amounts of habituation occurring on Trial 1 vs. Trial 2 as a function of trial spacing, presence of the dishabituator, etc.). Another possibility is that differences in long-term habituation were blocked by the appearance of fear on the 24-h delayed test trial, as evidenced by the increased number of boluses on such trials. Several facts argue against this interpretation. Activity was not completely suppressed on the delayed test trials, so that a floor effect was not present. Also, the test trials were sensitive to some of the experimental manipulations, such as the number of prior exposures to a particular maze (Experiments 3 and 4). Finally, it may be noted that the correlation between gross activity and the index of fear (number of boluses) was weak (Experiment 2).

The present short-term results are consistent with the hypothesis of the effects of self-generated priming on habituation (Wagner, 1976). According to this view, a stimulus will be less effective in eliciting an unconditioned or orienting response if the stimulus has been primed by the representation in STM of an earlier presentation of that same stimulus. In the present case, less exploration of the maze occurred when the prior exposure took place shortly before, which is consistent with the view that priming depends upon short-term memory. The decrement was stimulus specific, as would be expected if the priming effect depended upon a "match" between the contents of STM and the external stimulus. And the decrement could be removed by interpolation of a novel stimulus between the two massed repetitions of the target stimulus, as if the dishabituator was acting to clear the priming representation of the first stimulus presentation from STM.

The appearance of long-term (i.e., Trial 1-test trial) habituation agrees with the hypothesis of retrievalgenerated priming (Wagner, 1976). In this form of priming, the stimulus is primed indirectly through exposure to an associated stimulus, which acts to retrieve representation of the target stimulus from long-term memory into STM. In the present case, contextual cues and handling stimuli may have become the retrieval cues signaling placement in the maze. On the test trial, such cues could prime representation of the maze and thus lead to less exploration. (Thus, evidence of conditioning is shown by a diminution in the magnitude of the unconditioned response, e.g., Kimble \& Ost, 1961.) This hypothesis is supported by the findings of long-term maze-specific habituation that is also a function of the number of prior maze exposures.
There are sources of difficulty with the priming interpretation for the present studies. One is that the short intertrial interval used here, $5 \mathrm{~min}$, exceeds most estimates of the duration of representation in STM. Also, those factors that differentially affect the short-term response decrement should also affect the level of long-term habituation. The decrease in exploration with massed trials should be accompanied by a decrease in the joint processing of the contextual cues and maze stimulus representation, leading to a weaker association between the two sets of stimuli (see Terry, 1976; Terry \& Wagner, 1975; Wagner \& Terry, 1975, for discussion of the theory of priming and conditioning). Similarly, the posttrial dishabituators apparently did not interfere with the shortterm rehearsal necessary for long-term memory formation, even though short-term priming was disrupted. Finally, pretest exposure to novel stimuli did not reduce the effectiveness of the supposed retrieval cues to promote recognition.

One resolution of these problems is to suggest that all of the intertrial habituation observed was retrieval generated, i.e., was due to associations formed on Trial 1 and evidenced on Trial 2 and the test trial. Retrieval from long-term memory could reasonably be affected by the time since the previous exposure, the presence of novel stimuli at the time of retrieval, and the amount of training prior to testing. The fact that dishabituation occurred on Trial 2 but not on the test trial might have been due to the additional maze exposure received in the latter case prior to testing retention.

Alternatively, one might argue that performance on Trial 2 was affected by both self- and retrievalgenerated priming while the test trial was only affected by retrieval-generated priming, with certain of the present manipulations affecting STM (e.g., trial spacing and presence of a dishabituator). Finally, one could consider the possibility that a 24 -h delayed test was simply less sensitive than an earlier test to the effects of some of the prior manipulations. This view is supported by the marginal difference found after a 1-h delay (Experiment 1), and by an earlier report of smaller differential effects with a 24-h delay than with a 1-min delay (Davis, 1970).

The notion that associative learning underlies the present results may also explain certain discrepancies between these and prior studies. For example, withinsession decrements in activity have sometimes been reported without any apparent between-session retention of habituation. This could be due to the use of procedures that minimized conditioning to contextual stimuli, such as the use of detention boxes between trials rather than the more elaborate handling that occurred here (Montgomery, 1951, 1952) or by neutralizing such cues by prior exposure (Bronstein, 1972a, 1972b). 
The present findings readily fit within other theoretical frameworks that address trial-spacing effects, such as the models of stimulus fluctuation (Estes, 1955) or encoding variability (Martin, 1972). According to these models, stimulus recognition would depend upon the particular encodings of the target stimulus across successive presentations. A stimulus would be better recognized when a second presentation immediately followed the first, since the stimulus would be similarly encoded on both trials. However, long-term retention would be facilitated by encoding the stimulus in several different contexts, thus increasing the number of retrieval cues. This could be accomplished by lengthening the intertrial interval during training, or by placing "distractor" items between successive presentations (e.g., Bjork \& Allen, 1970). It should be noted that the present Experiment 4 is, in fact, modeled after a study of human verbal memory by Bjork and Allen (1970). These investigators varied the difficulty of distractor activity between two presentations of a to-be-remembered item. While a control study showed that the difficult distractor would reduce recall when it followed a once-presented item (like the present Trial 2 results), retention following the second presentation was actually better when the distractor was interpolated between the two presentations of the target (comparable to the present test trial results). These authors suggested that the disruptive effect of the distractor on immediate retention of the first presentation of the item was compensated for by the different encoding of the item's repetition. A similar interpretation may also apply to the present situation.

The above discussion of the habituation of locomotor activity has not addressed the apparent fear (evidenced by the increased number of boluses) on the test trials when a long retention interval was employed. One theory that is available to account for both fear and exploration is that of Montgomery (1955). According to this theory, exposure to a novel environment elicits both an exploratory drive and fear of the novel stimuli. Whether exploration occurs or not depends upon the strength of the fear aroused by the stimuli. For example, Montgomery (1955) demonstrated more exploration of an enclosed maze than of an elevated, open maze and suggested that fear predominated over the exploratory drive in the latter situation. In support of this view, exploration of the open maze increased with continued exposure to it, presumably as the fear habituated.

In the present situation, it could also be the case that a response tendency both of exploration and of fear were aroused, but fear was initially the weakest. Indeed, informal observations across these several studies indicated little hesitancy to begin traversal of the maze on the first trial. However, if the tendency to explore habituated faster than the fear, or if habit- uated fear showed more recovery from habituation over trials, then the fear drive might come to dominate on the test trials. Possibly, further trials beyond the test trial would indicate a recurrence of activity as the fear once again habituated.

While theoretical interpretation of the present findings remains open, empirically a rather stable pattern of data has been demonstrated. The results are consistent with Whitlow's (1975) observations concerning short-term habituation, and with Davis' (1970) studies of both short- and long-term habituation. However, the combined pattern is somewhat more complicated than expected from either of the above noted investigations. In general, the present results suggest that the study of exploratory activity does seem to be a viable preparation with which to investigate habituation.

\section{REFERENCES}

BerLyNe, D. E. The arousal and satiation of perceptual curiosity in the rat. Journal of Comparative and Physiological Psychology, 1955, 48, 238-246.

Berlyne, D. E. Conflict, arousal, and curiosity. New York: McGraw-Hill, 1960.

Bjork, R. A., \& Allen, T. W. The spacing effect: Consolidation or differential encoding. Journal of Verbal Learning and Verbal Behavior, 1970, 9, 567-572.

Bronstein, P. M. Open-field behavior of the rat as a function of age: Cross-sectional and longitudinal investigations. Journal of Comparative and Physiological Psychology, 1972, 80, 335-341. (a)

Bronstein, P. M. Repeated trials with the albino rat in the open field as a function of age and deprivation. Journal of Comparative and Physiological Psychology, 1972, 81, 84-93. (b)

Candland, D. L., Culbertson, J. L., \& Mayer, R. S. Parameters affecting adaptation to and retention of open-field elimination in the rat. Animal Behaviour, 1965, 13, 46-51.

Collerain, I. Frustration odor of rats receiving small numbers of prior rewarded running trials. Journal of Experimental Psychology: Animal Behavior Processes, 1978, 4, 120-130.

Danziger, K., \& Mainland, M. The habituation of exploratory behavior. Australian Journal of Psychology, 1954, 6, 39-51.

Davis, M. Effects of interstimulus interval length and variability on startle-response habituation in the rat. Journal of Comparative and Physiological Psychology, 1970, 72, 177-192.

Dember, W. N., \& KLeinman, R. Cues for spontaneous alternation by gerbils. Animal Learning \& Behavior, 1973, 1, $287-289$.

Estes, W. K. Statistical theory of distributional phenomena in learning. Psychological Review, 1955, 62, 369-377.

Fowlen, H. Curiosity and exploratory behavior. New York: Macmillan, 1965.

GLANZER, M. Changes and interrelations in exploratory behavior. Journal of Coniparative and Physiological Psychology, 1961, 54, 433-438.

Kimble, G. A., \& Ost, J. W. P. A conditioned inhibitory process in eyelid conditioning. Journal of Experimental Psychology, 1961, 61, 150-156.

MARTIN, E. Stimulus encoding in learning and transfer. In A. W. Melton \& E. Martin (Eds.), Coding processes in human memory. Washington, D.C: Winston, 1972.

Montgomery, K. C. The relation between exploratory behavior and spontaneous alternation in the white rat. Journal of Comparative and Physiological Psychology, 1951, 44, 582-589. 
Montgomery, K. C. Exploratory behavior and its relation to spontaneous alternation in a series of maze exposures. Journal of Comparative and Physiological Psychology, 1952, 45, 50-57.

MONTGOMERY, K. C. Exploratory behavior as a function of "similarity" of stimulus situations. Journal of Comparative and Physiological Psychology, 1953, 46, 129-133.

Montgomery, K. C. The relation between fear induced by novel stimulation and exploratory behavior. Journal of Comparative and Physiological Psychology, 1955, 48, 254-260.

Montgomery, K. C., \& Monkman, J. A. The relation between fear and exploratory behavior. Journal of Comparative and Physiological Psychology, 1955, 48, 132-136.

Parsons, P. J., Fagan, T., \& Spear, N. E. Short-term retention of habituation in the rat: A developmental study from infancy to old age. Journal of Comparative and Physiological Psychology, 1973, 84, 545-553.

Rubel, E. W., \& Rosenthal, M. H. The ontogeny of auditory frequency generalization in the chicken. Journal of Experimental Psychology: Animal Behavior Processes, 1975, 1, 287-297.

Schneider, G. E., \& Gross, C. G. Curiosity in the hamster. Journal of Comparative and Physiological Psychology, 1965, 59, 150-152.

TERRY, W. S. Effects of priming unconditioned stimulus representation in short-term memory on Pavlovian conditioning. Journal of Experimental Psychology: Animal Behavior Processes, 1976, 2, 354-369.

TERRY, W. S. Spontaneous alternation behavior as a function of intertrial detention environment. Psychological Reports, 1978, 42, 627-631.

Terry, W. S., \& Wagner, A. R. Short-term memory for "surprising" versus "expected" unconditioned stimuli in Pavlovian conditioning. Journal of Experimental Psychology: Animal Behavior Processes, 1975, 1, 122-133.

Thomas, T. R. Retention of environmental habituation in rats as a function of the environment during the retention interval Animal Learning \& Behavior, 1974, 2, 267-270.

Thompson, R. F., \& Spencer, W. A. Habituation: A model phenomenon for the study of neuronal substrates of behavior. Psychological Review, 1966, 73, 16-43.

WAGNeR, A. R. Priming in STM: An information-processing mechanism for self-generated or retrieval-generated depression in performance. In T. J. Tighe \& R. N. Leaton (Eds.), Habituation: Perspectives from child development, animal behavior, and neurophysiology. Hillsdale, N.J: Erlbaum, 1976.

WAgner, A. R., \& Terry, W. S. Backward conditioning to a CS following an expected vs. a surprising UCS. Animal Learning \& Behavior, 1975, 3, 370-374.

WhitLow, J. W. Short-term memory in habituation and dishabituation. Journal of Experimental Psychology: Animal Behavior Processes, 1975, 1, 189-206.

\section{NOTE}

1. By equating the Trial 2-test interval at $1 \mathrm{~h}$, the Trial 1-test interval is confounded between groups. For Group 5 , this latter interval is approximately $70 \mathrm{~min}$, but for Group 60 , it is $125 \mathrm{~min}$. Another group, similar to Group 5, was also run in this study, receiving a $2 \mathrm{~h}$ delay from Trial 2 to the test trial. Subjects here showed a decline in activity from Trial 1 to test (mean transformed scores $=6.28$ and 4.68 squares crossed), which was comparable to that of Group 5 receiving only a 1 -h delay. However, this group unexplainably did not show much decrease in activity on Trial 2, 5 min after Trial 1 (mean score $=5.74$ ), thus jeopardizing interpretation of this control's data.

(Received for publication July 11, 1978; revision accepted February 12, 1979.) 